\title{
Atypical Presentation of Acute Myeloid Leukemia
}

\author{
Kavita Agrawal ${ }^{\mathrm{a}, \mathrm{c}}$, Levin Miles ${ }^{\mathrm{b}}$, Nirav Agrawal ${ }^{\mathrm{a}}$, Asim Khan $^{\mathrm{a}}$
}

\begin{abstract}
We present a case of a 48-year-old male who presented with worsening pleuritic chest pain for $2 \mathrm{~h}$. He also complained of fever, malaise, headache and severe neck pain. Electrocardiogram (ECG) showed ST segment elevation in leads I, II, aVL and $V_{5}$ with PR elevation and ST depression in aVR. On admission, troponin-I was $14.8 \mathrm{ng} / \mathrm{mL}$. Based on ECG changes, elevated troponin and family history of early coronary artery disease, the patient was emergently taken to cardiac catheterization lab. Angiography showed non-obstructive coronaries, mild hypokinesis of mid inferior and anterolateral wall with ejection fraction (EF) of $40-45 \%$. Based on above presentation and angiography findings, the diagnosis of acute myopericarditis was made. $\mathrm{He}$ was started on colchicine and ibuprofen. The other workup to determine etiology of myopericarditis was negative as shown below. Given the history of fever, headache and worsening neck pain, we also became suspicious of meningitis. Lumbar puncture was performed which was negative. On the day of admission, he was found to have blasts on complete blood count and peripheral smear. Bone marrow biopsy and flow cytometry confirmed the diagnosis of acute myeloid leukemia (AML). He received induction and salvage therapy. Repeat bone marrow confirmed complete remission and normal cytogenetics. Although pericardial or myocardial biopsies are unavailable for our patient, in the absence of other causes, it does appear that his acute myopericarditis was associated with AML.
\end{abstract}

Keywords: Myopericarditis; Acute; Myeloid; Leukemia; Atypical

\section{Introduction}

Acute myeloid leukemia (AML) can infiltrate nearly all organ systems including the heart. Much of the heart involvement was not detected until autopsies because, for unknown reasons, it rarely produced detectable signs or symptoms. The

Manuscript submitted January 6, 2018, accepted January 18, 2018

aDepartment of Internal Medicine, Overlook Medical Center, 99 Beauvoir Avenue, Summit, NJ 07901, USA

bepartment of Pathology, Overlook Medical Center, 99 Beauvoir Avenue, Summit, NJ 07901, USA

${ }^{\mathrm{c} C}$ Corresponding Author: Kavita Agrawal, Department of Internal Medicine, Overlook Medical Center, 99 Beauvoir Avenue, Summit, NJ 07901, USA.

Email: kaviagrawal89@gmail.com

doi: https://doi.org/10.14740/wjon1083w estimated incidence of AML presenting as pericarditis is $1-2 \%$ $[1,2]$. However, we could not find any reported case of AML manifesting as myopericarditis. We report here an interesting case of myopericarditis as an initial manifestation of AML which is a very rare phenomenon. Our case also highlights that it is critical to be aware of atypical presentations of AML because it can lead to timely diagnosis and treatment of this lifethreatening condition.

\section{Case Report}

A 48-year-old Caucasian male presented to our hospital emergency room with the chief complaint of chest pain for $2 \mathrm{~h}$. Until two days prior to presentation, the patient had been in his usual state of health. At that time he noticed malaise, headache and fever. He also noticed severe right-sided posterior neck pain which was sharp and non-radiating. He had not noticed numbness, tingling, motor or sensory deficits. A day prior to presenting at our hospital, he had visited a nearby emergency room with complaints as above. His symptoms had been attributed to viral infection. He was given symptomatic treatment and was discharged home. However, in the next $24 \mathrm{~h}$, he had worsening symptoms. Two hours prior to presentation, he had sharp constant substernal chest pain that began at rest. The pain was aggravated by inspiration and being supine and relieved by lying in lateral position. The pain progressively worsened over the course of $2 \mathrm{~h}$ and was associated with mild shortness of breath. The pain was non-radiating and unrelated to exertion. He denied nausea, vomiting, photosensitivity, sick contacts or recent travel.

He did not have past medical history. He had past surgical history of umbilical herniorrhaphy. He had seasonal allergies. He was using testosterone supplements for bodybuilding. He denied smoking, alcohol or recreational drug use. His family history included myocardial infarction in the father who died at age of 52. He worked as an environmental constructor and in wooded areas which made him susceptible to tick exposure. He lived with his wife and was independent in activities of daily living.

Physical examination revealed a muscular male in mild distress. His temperature was $103.4^{\circ} \mathrm{F}$, pulse 100 beats per minute, blood pressure $100 / 66 \mathrm{~mm} \mathrm{Hg}$, respiratory rate 20 breaths per minute and oxygen saturation $94 \%$ on room air. His body mass index was $31.6 \mathrm{~kg} / \mathrm{m}^{2}$. Oral mucosa was dry. No lymphadenopathy was noted. Lungs were clear to auscultation bilaterally. Heart sounds, rate and rhythm were regular. No murmur or friction rub was heard. No reproducible chest pain was noted. Abdomen was soft, non-tender and non-distended 


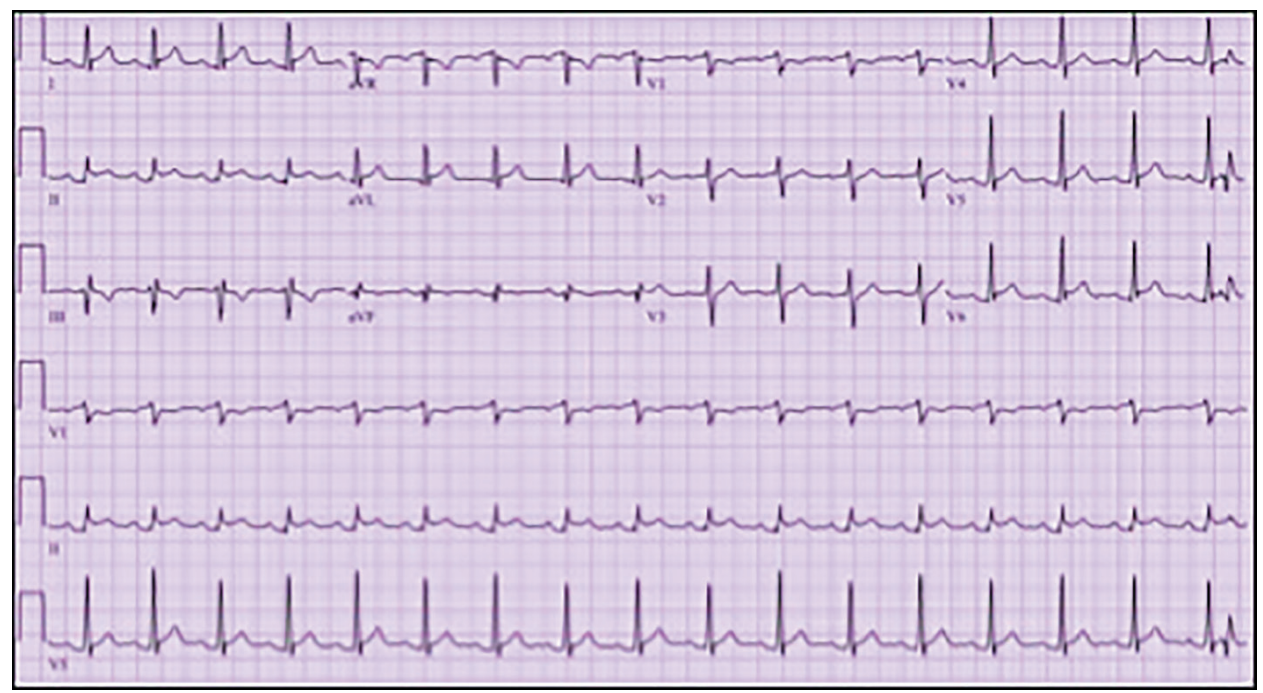

Figure 1. Electrocardiogram (ECG) showing sinus tachycardia, rate 103 beats per minute, ST segment elevation in leads I, II, aVL and $V_{5}$ with PR elevation and ST depression in aVR.

with no hepatosplenomegaly. Cranial nerves 2-12 were grossly intact. Motor strength and sensation in all four extremities were intact. On exam, there was no neck rigidity. Brudzinski's and Kernig's sign were negative. No rash or joint swelling was noted. No bruising, petechiae or history of easy bruising was present.

On admission, electrocardiogram (ECG) showed sinus rhythm, rate 103 beats per minute, ST segment elevation in leads I, II, aVL and $\mathrm{V}_{5}$ with PR elevation and ST depression in aVR (Fig. 1). Troponin-I was $14.8 \mathrm{ng} / \mathrm{mL}$. Complete blood count revealed a hemoglobin of $10.8 \mathrm{~g} / \mathrm{dL}$, white cell count of $13,000 / \mu \mathrm{L}$, blasts of $56 \%$, absolute neutrophil count of $1,430 /$ $\mu \mathrm{L}$ and platelet count of $83 \times 10^{3} / \mu \mathrm{L}$. Liver function tests showed alanine aminotransferase of $49 \mathrm{U} / \mathrm{L}$ and mildly elevated aspartate aminotransferase of $77 \mathrm{U} / \mathrm{L}$. Patient was tested for Lyme disease, anaplasmosis and ehrlichiosis which was negative. Blood cultures were negative. Other investigations are mentioned in Table 1 below.

Given the patient's family history of early coronary artery disease, use of testosterone supplements and ECG abnormalities, he was diagnosed with ST elevation myocardial infarction. He underwent emergent coronary angiography. Angiography did not reveal any coronary artery disease. It showed mild left ventricular systolic dysfunction with ejection fraction of 40$45 \%$. There was mild hypokinesis in the mid inferior and mid anterolateral walls and no pericardial effusions. Transthoracic echocardiography (TTE) revealed same findings as above. In light of the angiographic and echo findings, his cardiac diagnosis was changed to acute myopericarditis. The patient was started on colchicine $0.6 \mathrm{mg}$ two times a day and ibuprofen $600 \mathrm{mg}$ three times a day. Furthermore, an extensive workup was performed to determine the etiology of myopericarditis. The workup, as shown in Table 1, was negative.

Given the patient's fever, headaches, severe neck pain and susceptibility to tick exposure, there was a suspicion of meningitis. The patient was started empirically on intravenous ceftriaxone and vancomycin. Lumbar puncture was performed on the day of admission. Cerebrospinal fluid (CSF) cell count, glucose and protein were within normal limits. CSF culture was negative. Other organisms tested for meningitis/encephalitis as shown in Table 2 were all negative.

Given the abnormalities noted in the patient's initial CBC, the peripheral smear from admission was examined. Red blood cells (RBCs) showed moderate anisopoikilocytosis with preserved central pallor. No schistocytes or nucleated RBCs were seen. There was a paucity of platelets. White blood cells were largely represented by large irregular basophilic cells with large nuclear to cytoplasmic ratio and eccentrically placed irregular nucleus (Fig. 2). The initial impression was AML.

On day 3 of hospitalization, a bone marrow aspiration and biopsy were performed. Pathologic examination showed hypercellular marrow with diffuse mononuclear infiltrates representing $>95 \%$ of marrow cellularity. The infiltrates were composed of blasts cells with high nuclear to cytoplasmic ratio and

Table 1. Workup for Myopericarditis

\begin{tabular}{ll}
\hline Human immunodeficiency virus (HIV) & Not detected \\
Hepatitis A & Not detected \\
Hepatitis B surface antigen & Not detected \\
Hepatitis C antibody & Not detected \\
Cytomegalovirus & Not detected \\
Coxsackie type A and B antibody & Not detected \\
Influenza A and B & Not detected \\
Parainfluenza 1-4 & Not detected \\
Adenovirus & Not detected \\
Enterovirus & Not detected \\
Mycoplasma pneumonia & Not detected \\
Antinuclear antibody (ANA) & Negative \\
Antineutrophilic cytoplasmic antibody (ANCA) & Negative \\
\hline
\end{tabular}


Table 2. Meningitis Panel in Cerebrospinal Fluid

\begin{tabular}{ll}
\hline Listeria monocytogenes & Not detected \\
Neisseria meningitidis & Not detected \\
\hline Enterovirus & Not detected \\
\hline Herpes simplex virus 1 and 2 & Not detected \\
Escherichia coli K1 & Not detected \\
\hline Hemophilus influenza & Not detected \\
Cytomegalovirus & Not detected \\
Streptococcus agalactiae & Not detected \\
Human herpesvirus 6 & Not detected \\
Human parechovirus & Not detected \\
Varicella zoster virus & Not detected \\
Cryptococcus neoformans & Not detected \\
\hline
\end{tabular}

conspicuous nucleoli. Residual hematopoiesis was scant (Fig. 3). Flow cytometry of the bone marrow aspirate was consistent with AML (Figs. 4 and 5). Fluorescence in situ hybridization (FISH) analysis was positive for $\mathrm{t}(6 ; 9)(\mathrm{p} 23 ; \mathrm{q} 34)$. The rearrangements of $6 \mathrm{p} / 9 \mathrm{q}$ are seen in myeloid neoplasms, including AML. Based on the bone marrow evaluation, the diagnosis of AML was confirmed.

On day 3 of hospitalization, the patient continued to have a fever of $101^{\circ} \mathrm{F}$. Repeat complete blood count showed an absolute neutrophil count of $780 / \mu \mathrm{L}$. Therefore, ceftriaxone was changed to cefepime for neutropenic fever. He was continued on vancomycin.

On day 5 of hospitalization, he was transferred to a tertiary care center for the treatment of AML. On the day 7 from initial presentation, he was started on induction therapy. This involved continuous 7 days intravenous infusion of cytarabine with a short intravenous infusion of idarubicin on the first 3 days. One week after the completion of induction therapy, a bone marrow biopsy was done. It showed $10-20 \%$ residual AML. He was given salvage therapy with high-dose cytarabine (HiDAC) and midostaurin. The patient tolerated the chemotherapy well. Repeat bone marrow biopsy confirmed complete remission with normal cytogenetics. As of the writing of this

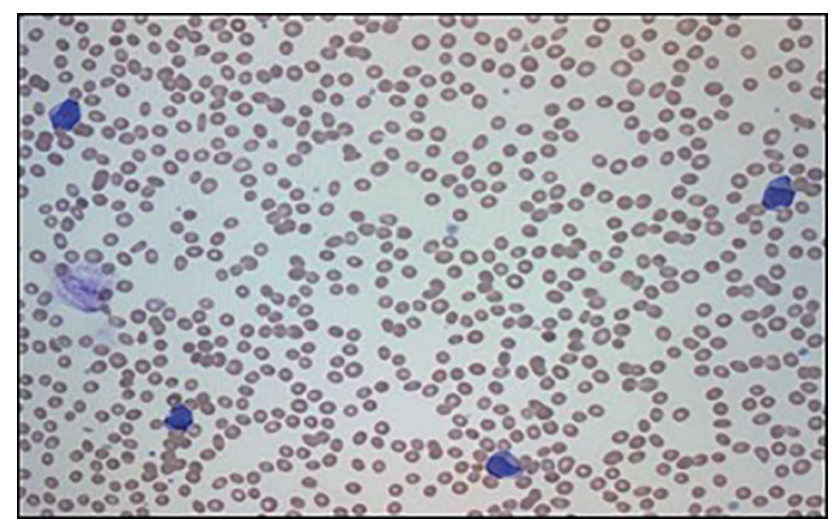

Figure 2. Peripheral smear showing increase in myeloid precursors with blasts.
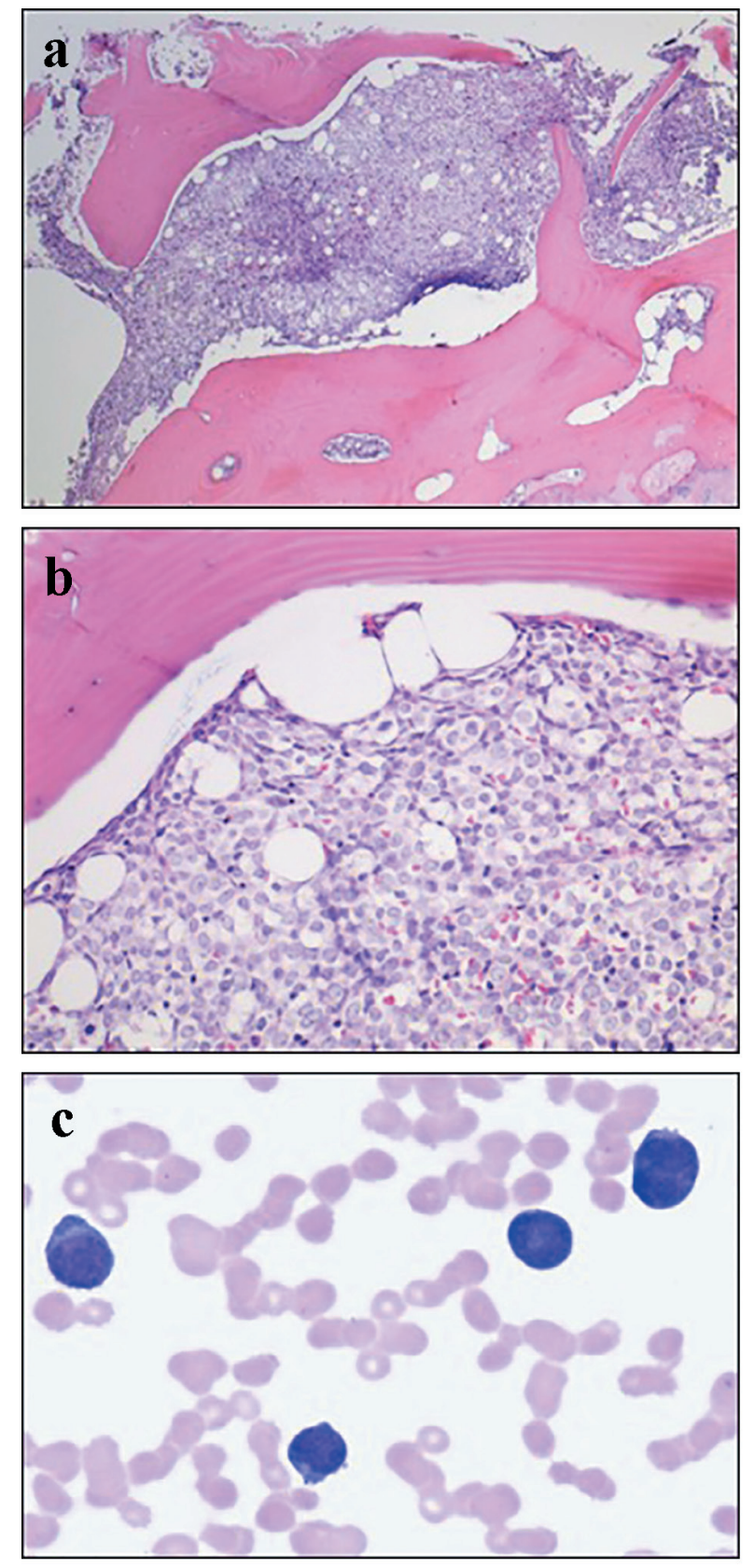

Figure 3. (a) Low power bone marrow $(H \& E, \times 40)$ demonstrating a packed marrow. (b) High power bone marrow (H\&E, × 200) showing cellular infiltrates with high nuclear/cytoplasmic ratio and prominent nucleoli consistent with myeloblasts. (c) High power bone marrow aspirate with Giemsa-Wright stain $(\times 600)$ showing myeloblasts.

case, the patient is awaiting HiDAC consolidation treatment and bone marrow transplantation.

\section{Discussion}

AML is a condition characterized by clonal proliferation of myeloid precursors with loss of ability to differentiate into ma- 

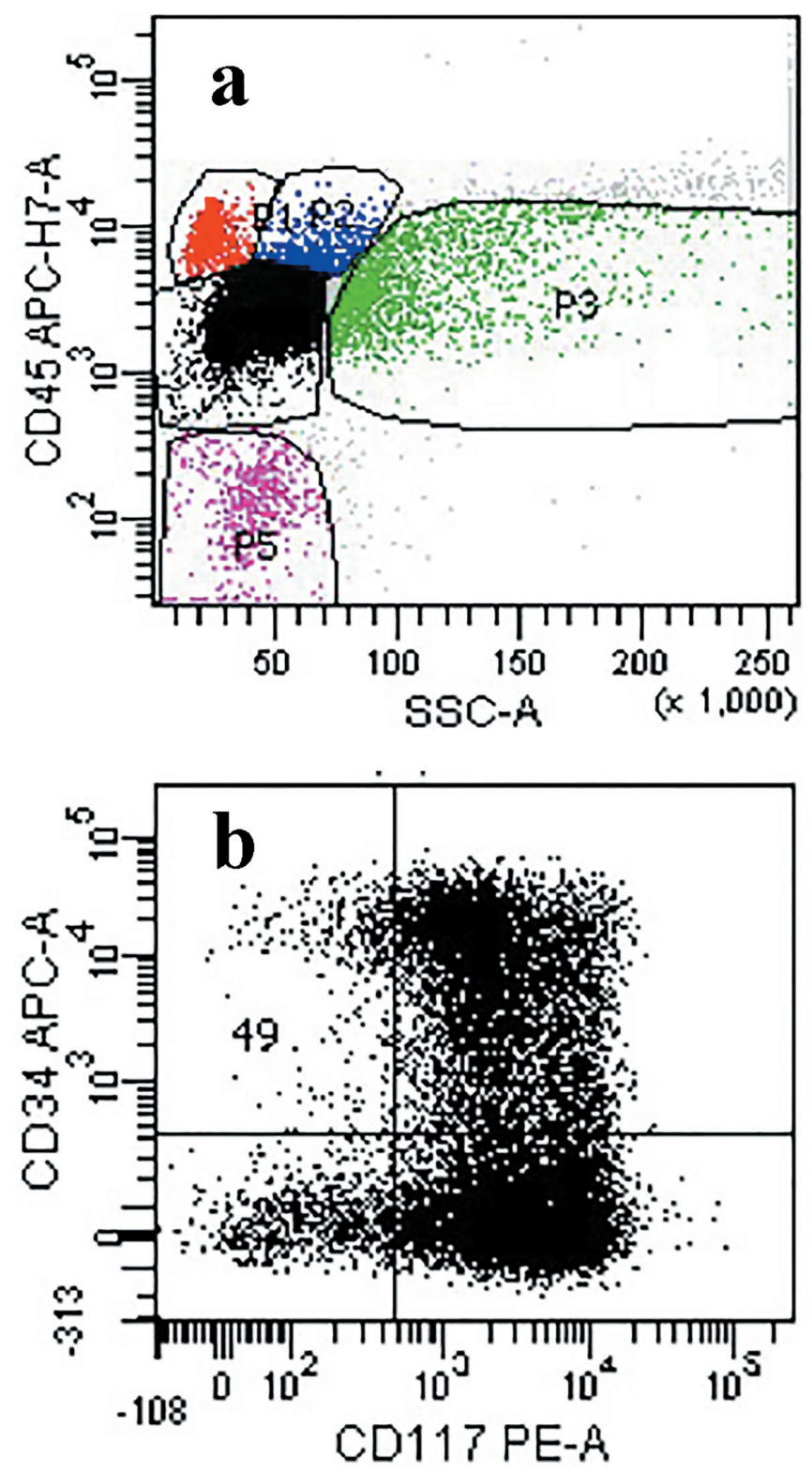

Figure 4. (a) Flow cytometry of the ungated scattergram plot of the marrow aspirate showing a high proportion of events in the dim CD45/ low side scatter region (black dots). (b) Blast gate showing expression for CD117 and partial expression for CD34 consistent with blasts.

ture forms. It can manifest clinically with variable symptoms. The typical presentation involves symptoms related to anemia, thrombocytopenia and/or leukopenia. This includes fatigue, weakness, increased bleeding tendencies or predisposition to infections.

It is also important to be aware of the atypical manifestations of AML. This can prompt thorough search into this lifethreatening condition. Skin can be involved in up to $10 \%$ of AML cases [3]. Skin lesions can manifest as mass-like nodules, and/or erythematous or as a violaceous papules or plaques. Other uncommon manifestations include vitiligo, stasis dermatitis or erythema nodosum [4-6]. In AML, skin manifestations are indicative of aggressive disease and poor prognosis [4]. AML can present with joint manifestations in $4 \%$ of the cases [7]. This includes bone pain/tenderness, arthralgias and symmetric or migratory polyarthritis. The presence of leukemic synovitis is indicative of systemic involvement and should prompt aggressive treatment [7]. Central nervous system involvement is very unusual with estimated incidence of less than $5 \%[8,9]$. The symptoms include headaches, cranial nerve palsies, altered mental status or seizures. AML can manifest as myeloid sarcoma in less than $1 \%$ of the cases [10]. Myeloid sarcoma is a solid tumor composed of myeloblasts at an extramedullary site. The most frequently affected sites are skin, lymph nodes or bone $[11,12]$. However, any part of the body can be affected.

The involvement of heart during the course of AML is not infrequent. Robert et al studied a large case series involving 420 autopsies of leukemic patients, either myelogenous or lymphocytic, from the year 1954 to 1964 [13]. The results showed $288(69 \%)$ patients suffered from some degree of involvement of the heart such as direct leukemic infiltration or localized hemorrhages or both [6]. The most common cardiac site of involvement was the pericardium, affecting $190(46 \%)$ patients followed by left ventricle, right ventricle and left atrium. Much of the heart involvement was not detected until autopsies because, for unknown reasons, it rarely produced detectable signs or symptoms. An initial manifestation of AML as pericarditis is very infrequent. The exact incidence is unknown, but is estimated to occur in $1-2 \%$ of the AML cases. In the above case series, only nine of 420 patients $(2.1 \%)$ presented with chest pain typical of pericarditis [1]. Kulkarni et al studied 80 cases of leukemia in the course of 6 years and only one case had pericarditis as the initial presentation [2]. However, based on our literature review, we could not find any reported cases of AML presenting as myopericarditis.

The patient presented with symptoms of pleuritic chest pain and dyspnea, ECG changes, elevated troponins and presumed new focal left ventricular dysfunction on echocardiography that lead to the diagnosis of probable myopericarditis. The workup to determine the etiology of myopericarditis including serologies for bacterial and viral illnesses as well as cardiac catheterization was unrevealing. The definitive diagnosis of the myopericarditis and associated etiology would have required an endomyocardial biopsy. But this is usually not recommended in patient with no or mild left ventricular dysfunction (ejection fraction greater than or equal to $45 \%$ ), no signs of heart failure and arrhythmias [13]. Also, the presence of blasts on peripheral smear and bone marrow biopsy confirmed the diagnosis of AML. We determined the cause of his myopericarditis to be AML due to the close temporal association between the two and exclusion of other etiologies. The patient had resolution of chest pain and dyspnea upon treatment with colchicine and chemotherapy. As a result, endomyocardial biopsies, and their associated risks, were avoided in our patient. Our case highlights myopericarditis as the initial manifestation of AML.

\section{Conclusion}

Our report illustrates an unusual case of a 48-year-old male 


\begin{tabular}{|c|c|c|}
\hline \multicolumn{3}{|l|}{ Results } \\
\hline Antibody & Docription & Roults \\
\hline $\mathrm{CD} 2$ & Pan T-Cell Artigen, NK Cells & Nogtive \\
\hline $\mathrm{CD} 3$ & Pan T.Cell Asoigen TCR Complex & Negative \\
\hline CD4 & T-Helper Subce, MaHC-II co-roverece, dim in minosies & Positivo Dis \\
\hline CD5 & Pan T. cell moker, B cell bumboema subset & Ningtive \\
\hline CD7 & Pas-T Cell Arcigen, NK Cells, Early Myelois Cells & Positivo Din \\
\hline CD10 & 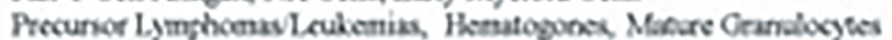 & Nogative \\
\hline CDilb & Moeoryter, Mfycloid Cell, NK Cell T and B Subues & Positive Din \\
\hline CD13 & Early and Mature Myeloid Cells and Mcnosics. LGL Subert & Positive Modenes \\
\hline $\mathrm{CD} 14$ & Moesytes: strong Alyoloid Cells: weik & Negative \\
\hline CDis & Myckid Cells asd Monocyes & Nogative \\
\hline CD16 & Mature Mfycloid Celks NK Colls & Nogative \\
\hline CD19 & Pan B-Celd Antigen; Weik of Nogtive in Phrem Cells & Nogative \\
\hline CD30 & Mature B-Cells & Novative \\
\hline $\mathrm{CD} 23$ & B-eell subset, CLLSU, follicular dentritic nestrocok & Nogative \\
\hline $\mathrm{CD} 33$ & 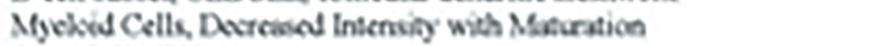 & Rositive Dis \\
\hline $\mathrm{CD}_{4}$ & Sten Cells Blasts & Positive Msulerase \\
\hline CD33 & Progenitce Lymghoid Calls, Btasts Plasma Colls & Positiono Moderies \\
\hline CD45 & Pan-louborye Antion & Positive Moderas \\
\hline CD56 & NK Cells & Nogative \\
\hline CDS4 & Mcencytes and Earty Lineuge Mfyolotd Cells & Posituvo Din \\
\hline CD71 & 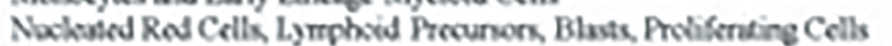 & Positivo Din \\
\hline CD 117 & e-kis & Positivo Din \\
\hline HA.DR & D. 2 Recoptor, Ativatad T and B Cells, Hairy Cells & Positive Msolenes \\
\hline supra & B Lymposites & Nozative \\
\hline
\end{tabular}

Figure 5. Flow cytometry acute myeloid and lymphoid analysis: marrow aspirate with immunophenotypic profile consistent with involvement by AML (non-M3 phenotype).

presenting with myopericarditis as an initial manifestation of AML. The awareness of atypical presentations can lead to timely diagnosis and prompt treatment of this life-threatening condition. Cases have been reported with AML presenting as pericarditis or with pericardial effusions. To our knowledge, this is the first reported case of AML presenting as myopericarditis.

\section{Conflict of Interest}

The authors declare that there is no conflict of interest regarding the publication of this paper.

\section{Disclosure}

The above case has been presented as an e-poster at Hematology and Oncology Conference.

\section{References}

1. Roberts WC, Bodey GP, Wertlake PT. The heart in acute leukemia. A study of 420 autopsy cases. Am J Cardiol. 1968;21(3):388-412.

2. Kulkarni AG, Patil AP, Desai SR. Pericardial involvement as a presenting feature of acute myeloblastic leukemia. Brief communication. J Assoc Physicians India. 1992;40(5):345-346.
3. Ratnam KV, Khor CJ, Su WP. Leukemia cutis. Dermatol Clin. 1994;12(2):419-431.

4. Kaddu S, Zenahlik P, Beham-Schmid C, Kerl H, Cerroni L. Specific cutaneous infiltrates in patients with myelogenous leukemia: a clinicopathologic study of 26 patients with assessment of diagnostic criteria. J Am Acad Dermatol. 1999;40(6 Pt 1):966-978.

5. Newman MD, Milgraum S. Leukemia cutis masquerading as vitiligo. Cutis. 2008;81(2):163-165.

6. Papadavid E, Panayiotides I, Katoulis A, Pappa V, Dervenoulas I, Stavrianeas N. Stasis dermatitis-like leukaemic infiltration in a patient with myelodysplastic syndrome. Clin Exp Dermatol. 2008;33(3):298-300.

7. Avina-Zubieta JA, Galindo-Rodriguez G, Lavalle C. Rheumatic manifestations of hematologic disorders. Curr Opin Rheumatol. 1998;10(1):86-90.

8. Cheng CL, Li CC, Hou HA, Fang WQ, Chang CH, Lin CT, Tang JL, et al. Risk factors and clinical outcomes of acute myeloid leukaemia with central nervous system involvement in adults. BMC Cancer. 2015;15:344.

9. Bar M, Tong W, Othus M, Loeb KR, Estey EH. Central nervous system involvement in acute myeloid leukemia patients undergoing hematopoietic cell transplantation. Biol Blood Marrow Transplant. 2015;21(3):546-551.

10. Dores GM, Devesa SS, Curtis RE, Linet MS, Morton LM. Acute leukemia incidence and patient survival among children and adults in the United States, 20012007. Blood. 2012;119(1):34-43.

11. Campidelli C, Agostinelli C, Stitson R, Pileri SA. Myeloid sarcoma: extramedullary manifestation of myeloid 
disorders. Am J Clin Pathol. 2009;132(3):426-437.

12. Pileri SA, Ascani S, Cox MC, Campidelli C, Bacci F, Piccioli M, Piccaluga PP, et al. Myeloid sarcoma: clinicopathologic, phenotypic and cytogenetic analysis of 92 adult patients. Leukemia. 2007;21(2):340-350.

13. Cooper LT, Baughman KL, Feldman AM, Frustaci A,
Jessup M, Kuhl U, Levine GN, et al. The role of endomyocardial biopsy in the management of cardiovascular disease: a scientific statement from the American Heart Association, the American College of Cardiology, and the European Society of Cardiology. Circulation. 2007;116(19):2216-2233. 